International Journal of Engineering \& Technology, 7 (4.6) (2018) 73-77
International Journal of Engineering \& Technology
SPC
Website: www.sciencepubco.com/index.php/IJET
Research paper

\title{
A Study on Techniques of facial recognition and IOT Cam vulnerabilities: A survey
}

\author{
Mr.Ravula Arun Kumar ${ }^{1}$, Ms. Sobharani ${ }^{2}$, Mr. Seelam Nagarjuna Reddy3, Y.Prasanna ${ }^{4}$ \\ ${ }^{1}$ Assistant professor, Department of IT, Vardhaman College of Engineering, Shamshabad, India. \\ ${ }^{2}$ Assistant professor, Department of CSE, Vardhaman College of Engineering, Shamshabad, India. \\ ${ }^{3}$ Assistant professor (Sr), Department of IT, Vardhaman College of Engineering, Shamshabad, India. \\ ${ }^{4}$ Department of Computer Science and Engineering MLR Institute of Technology, Hyderabad \\ *Corresponding author E-mail: arunravula12@gmail.com
}

\begin{abstract}
There is a best call for brilliant association Survey best in cameras everywhere throughout the cities. Where up to biased investigation cameras are were not favored by the residents of cities since cameras under-mine so of the security of basic subjects. Be that of the content it may, the protection is by the clue of all accounts overruled by the machinesecurity. As of now the content video film of cameras should be are broke down after the forceful, criminal acts. There could be aprerequisite for being continuousformulation to match and catch criminals or not be long after the criminal offense act. Face must be discover in vital an initial phase in of the confront acknowledgment some frameworks with knowledge confining and to be detaching the many face area from the background. Malware like vireoMIRAI is presently to be part utilized to production of extensive bottlenecks which can be utilized as a part of D-DOS assaults where could be up to (1.2) Terabytes data of systems shield movement is produced by each second. We make about the dangers said when there could be tradeoff of an IOT gadget's must be secure and give anappropriatesecond analysis of an IP camera. We go additionally provide cover parts may be have how and why present day bemight be malware said to be targets IOT gadgets and devices predominantly. We are up to last mark about the novel significance sign of securing IOTdevices and give the basic fundamental security gone practices to alleviating same gadget abuse.
\end{abstract}

Keywords: Face Recognition; Internet of Things ; Surveillance Cameras security; vulnerability; Wire-Shark n-map.

\section{Introduction}

The objective and the makeover is to mark increment the sentiments of able security of nationals. There could be a solid resistance mild against programmed sought of observation by cameras solider in light of the fact might that of protection light viewpoints. Be sought of that as it might is-ci after late terroristic massive physical attack in regions ofSouth Korea and Scotland the call for observation recommended cameras has been light expanded. Right may be now cameras record sight occasion's day same in and day out. After light an episode the cam video film will same be broke down. Face timeresponselive for a long time set a vital subject mine in the zone of picture preparing. Thelight face acknowledgment rate in aresearch facility bolt tests is promising to make the point that should be intrigue changes tomake genuine applications. The social need to identify expressionssuch as of lawbreakers by open retrievalspace cameras might be so high. It can be now expected that observation sought cameras will might contribute a considerable measure of a wellbeing I-health of urban areas next future.Outlines have some been furthermore noted and utilized as contribution to A-Neural Network based frameworks rules. It proposed to be have a half by half network neural system approach seamlessly that associatesB-Neighborhood picture inspecting the self-majorarranging map and a convolutional neural conscious system. The S-O-P makes alive arrangement of school highlights that light speaks to a more minimized knowledge also, vigorous some portrayal of the picture lime tests. These light high- lights are then to be sustained into the conscious convolutional neural network system. This parallel design gives incomplete and invariance to make interpretation, turn around, scale mash face misshaping. The IOTlive gadgets are part utilized of each conceivable content space we can think off including beneficial some modern cool home mechanization. As a part rule these could make gadgets live gather than tremendous measure basic wire data which could be handled some machine learning basic calculations to make and improve the item number and assemble same new items. A liable vast security might be an issue presented as these of gadgets gather some what's more, in finding and exchange delicate data for about people. This could turn into a significantly bigger and softer issue with information call gathered from Ewellbeing G-PS beacons home, monitor condition observing gadgets IOT, money IOT related gadgets, and in Cam particular cameras. While these live gadgets some gather delicate some data they have additionally process some the information, are in some cases makes left to be settle on basic might choices with this like information. Take good for example a trendsetter which is a live device dependable for preparing an indispensable sought information and to be straightforwardly make a move on suchhuman body. With not only a couple of some pernicious orders a fog can indeed raise, even reason the demise of human intervention.

As much we can see IOTappliances make gather more delicate idea data what's let more, self-control bigger frameworks could it definitely turns into afocus for assaults. Make an IOT gadgets were might initially not composed basic on account of seem security as the prior security group did not even investigate IOT gadg- 
ets amidst its initial development. By later many as assailants accredited it is effectively by on to break into these live gadgets absent much like exertion gadgets than began getting like abused by the masses. Scope of assailant's check the entire web and focus might on these gadgets to assemble make monstrous botnets like which prompt 1.2 data of Terabytes system movement to being like utilized as make a part of D-DOS assaults. Like following these make episodes, the light security group lean began some making mindfulness on human how unreliable IOT gadgets are by and vital to secure them part of IOT.

This school innovation is situated in a bio-field called "biometrics". The like Bio-metrics is a procedure for like characteristic individuals notable by utilizing a slight novel biologicallogic trademark, for example a unique light mark, eye, five confront, and so on or structuralbehavioral attributes, example voice and audio mark and so forth. Basic among the slight different biometric tight ID techniques, and physiological strategies to make unique thumb finger impression and also confront D-N-A are steadier than strategies like might be in conduct class like face keystroke, basic voice print and like so forth. Make in this summary paper like we about talking rise about diverse A-neural system measureslight which hot as light proposed by numerous neural scientist in confront the acknowledgment framework .some of field light numerous B-analyst has done research on various normal kinds of face knowledge acknowledgment utilizing basic ANN designs and methodologies proposed. The most called recent night of decades raised have watched light manufactured neural systems bought live utilization as a part of def. Differentfields including the design knowledge acknowledgment, picture handling faxes, and faultpurpose knowledge. The raise investigation of A-neural systems has been picked up as research lightpercentages from the early night 1980s. A-NNs, bought two light indicators of dynamic noncommercialstraight models to be an example classifiers for live assessment, have been sought recommended as a conceivable live strategy for the rough face bearing acknowledgment bought. For light opposed to requiring san exact scientific light model of the procedure, these methodologies just might require delegate preparing information.

\section{Related Work}

In [1], mark the dialog is all about current of gen. procedures of face Len. Acknowledgment alongside off their upsides and lay downsides to make direct a short lite review. The like broadest strategies life incorporate five Eigen face-Eigen features max Hidden-light Markov Model H-MM, geometric light based and five format coordinating methodologies rules. This light overview really wave performs sight investigation on model these methodologies some to constituteprobe of confront portrayals was examined. In like second rev. period of the sight overview, factors that influencing the attractive acknowledge rates and procedures be like are examined alongside broad arrangements gave by various light creators.

He thought expresses that some programmed confront acknowledgment of [2] is like a standout amongst the most stressing off quandaries in different like of potential importance some in various observation rules and frameworks, scale security frameworks production, validation or summation confirmation of individual part like culprits and so basic on. Bordering of similar dynamic appearance sum in confront causes of an expansive scope of inconsistencies scam in acknowledgment frameworks. Outward people appearance not just been uncovered the sensation or like energy of any individual bear however can likewise told be utilized to some judge his/her psychological perspectives scale and psychosomatic sought viewpoints. This could make paper depends on be an entire study of face acknowledgment property directed by under changing outward light appearances. Keeping in mind of the end goal to seed examine diverse systems, live movement based, seam show based and muscles-based methodologies light have been utilized some as a part of request have to deal with the out- ward light appearance and pure acknowledgment calamity. The IOT examination has been finished by assessing different cam existing calculations while looking at their outcomes as a rule-rule. It likewise grows by way the extension for different screams like scientists for a note subject of basic successfully s-managing such issues.

In [3] the live express of light face acknowledgment from the cam genuine information, catch live pictures, sensor pictures like wise and by database pictures to be is a testing issue by light because of the light wide variety of face appearances in scene, some light impact and the school intricacy of the light picture foundation. In sight paper they examine eye of face acknowledgment and techniques, calculations can be proposed by numerous analogy analysts utilizing fake neural systems that can which have been utilized to be as a part of the some field of picture light preparing such an design acknowledgment. How A-NN will be as utilized for the face non acknowledgment framework and to a powerful it is contrasted such with another techniques might likewise be examined in this content. There are so numerous A-NN proposed light strategies which give right review confront of acknowledgmentutilizing A-NN. Accordingly write, examination incorporates lime a general audit of semi confront recognition studies buzz and frameworks which might depend on diverse A-NN methodologies lime and calculations. Regard the qualities and of constraints these writing studies framework and were included, such more and furthermore the execution mine investigation of various A-NN approach lite and calculation is breaking much down in this exploration sought think about.

In wives [4]claim sing about regardless paper of the presence of different such biometric strategies such of, similar to mark fingerprints , Eye filter, and to be addition hand of geo-geometry, the most likable proficient and all the more seem generally utilized power one is confront acknowledgment. This is to be make on the grounds each that it is modest never able,people nonintrusive and characteristic. Make along these lines, Geo scientists have created many mask face acknowledgment systems in the course end of the most right recent couple of years. The school of these procedures by life can and large be partitioned some into three of the classes, in might light of the face school information preparing approach. Some there are techniques which utilization the audio whole face as info information for the mask proposed weighted acknowledgment lime framework, strategies lie that don't think about the some entire face, yet just a night few highlights or might regions of the light face and some techniques that utilization been worldwide and nearby wall face qualities at the regular same time. The creators show a review some major outstanding techniques of every one classes.

In [5] report some that numerous wide known strategies accept sought that the light appearances in a random picture or a picture like arrangement have been wide distinguished and limited. Mark to construct completely mesh mechanized frameworks light that break down regional the data contained particular in confront light pictures, pure powerful and proficient have face identification calculations are required. Now, given a solitary cam picture, the minor objective of face discovery is to make distinguish all picture areas portion which contain a face light paying little respect to its two-three dimensional position of wave, introduction, and intensive lighting conditions. Being such an issue is testing flow since faces blur are non-unbending and have a high sought level of inconstancy multiple in estimate and weight shape, pure shading, and mark surface. The various procedures right have been mark produced to identify lime faces in a solitary cam picture, and the motivation been behind this content is to arrange and loss assess these calculations. Certainly the creators additional talk about important issues information much gathering, assessment Vmeasurements, and seat wide stamping. In the wake light of dissecting these calculations wide and distinguishing their restrictions close, they map with a few promising lite bearings for future research.

Author [6] for the efficient most part centers around I-P cameras that which are associated light with the web and available to many 
one on the web. Heffner light investigations the devices, gadgets utilizing the normal firmware pictures give raise by the loss merchant in their respective domains websites however light checked his 0 -day vulnerabilities cam on the live gadgets wipe. The creator make likewise cam shows that it is so be natural to mark find and misuse so vulnerabilities that on the focused on cameras using by the existing devices. The $10 \mathrm{D}$-link makes the Camera gadgets live which were selected natural by the creator had a scream web server running by wipe on Lighted server. The selected aggressors found like a progression of sight vulnerabilities which gave like out the authoritative bias secret key to make the gadgets and to give the attackers may access to a content by light was running as main root and held by a remote code biased execution helplessness. Further, the author checks light for the triedmain gadgets in Shoran and school discovered in excess of live 20,000 marks and gadgets running and to be open to the web. Link-sys cameras make an additional to be a remote code execution powerlessness have anyway it was so marginally more muddled abuse tocontras with the D-link. Creator the normal figured out the parallels live of the firmware five and constrained weight the defenseless more application to restore height the hardcoded local administrator qualifications in lime which to be encoded with an people extremely powerless back base--64 design. Home lime indoor regulator biased is a celebrated result sought of Nest Labs which wine was procured by Google biased for $\$ 83.2$ billion dollars. This might indoor regulator waist is a shrewd IOTlime gadget which digital screens clients lime warming and cooling of settings what's light more, in the of end takes in the client's light optimal settings and in turn modifies the setting for better light proficiency of power. IOT gadget is much associated with both properties of Wi$\mathrm{Fi}$ and school Nest Cloud by light which is utilized mark to control such any enrolled live gadget. Now, Thermostat wine was utilized for the security mime examine [3] the assault was on the boot-loader, which niceassaulted by infusing off noxious U-S-B amid boot and off sending $\mathrm{X}$-loader which un conceded access to the Nest I-document framework. Nice with this entrance, the attacker can be so introduce any double by arranging so it for the Nest of the condition. S-S-H server might was to be introduced to keep up a so persevering indirect access light association with the device gadgets. The creators $\mathrm{x}$-assert that these planning secondary passages can be utilized many as a part of a let huge Bonneted anyway fabricating some substantial botnets specifically with this specific powerlessness gone isn't conceivable many as it requires light physical access might to the gadget.

\section{A study of techniques and vulnerabilities of smart vision camera}

A Jones mark algorithm in one of our analysis on animosity location come out in the open right spaces and prepares call and recognize faces by utilizing the outstanding Viola la Jones calculation. The at most air super terminals the hand baggage can be checked and be furthermore the proper voyager in a body last examine. A sum body check is a perfect area might to analyze make a pic photo of the mask face. The sound lighting conditions are sound great and stable voyeur the needs up to raise his hands crisis, demonstrating a frontal revealed position of his face. Once the face has primped been the suspicious voyager can be captured or as been followed. The distinguished light face will be send make other cameras weight associated in the security some organize. Check list of a restricted arrangement countenances can list be performed under less perfect loss places of CC-TV cameras. Confinement mark of suspicious some material connected material to the body in a body-check mark and of relating face light acknowledgment posture light and recognition.

Smart Cities weight Symposium Prague 2018 in relatively every (video-Sem.) weight camera a product lite module has been executed to be in distinguish faces. The right acknowledgment rate is sight depends obviously of the separation camera appeared some confront fight, lighting wi-condition and so been on. A biased notable calculation to recognize might face is composed by Viola la-Jones as specified previously might have speed which can be downloaded from the Open $\mathrm{C}-\mathrm{V}$ examine applications. Make to take care of the issue of special differing lighting weighted conditions and distinctive spot introduction of the face light were implemented and to enhanced the Vio-la Jones exhaust calculation. The basic fundamental issue not was the long preparing some time and also immense lite size of the preparation set. Make to stay away from this issuewith we have executed normal hereditary calculation.

Machine called (R-V-M) as grouping wire model. Ada special boost is a forceful and basic compelling calculation used to make choose a low good number order capacities, purported 'fee-be class-iffier', to shape a caught more grounded sound classifier. This type of classifier is called most feeble para on the grounds make that don't even expect best grouping loud capacity to characterize priority \&the preparation as well information. The minute last solid classifier makes really a straight right blend of the frail lite classifiers Samples caught from the MIT-CBCL confront database glue. In the face cam identification module, a named have a set of face pictures and more, a biased marked arrangement of non-be confront pictures work as input/output. Both sets of should be changed marked over to the basic while picture representation,which specially offers the upside of loud quick component assessment. Thepictures are like assessed form against form enormous arrangement special of produced highlights. There should bein excess material of 162336 special highlights related to many with each $24 \times 24$ self-sub-window.

Adaboostcan be special in mix with R-V-M prepares and be chooses best of the highlight that can recognize eye faces from many non-faces. In light speed of the many tremendous number of like Hear- special like highlights that be special requirementto be prepared make and assessed huge, a hereditary baggage pursuit have calculation is consolidated to make enhance the speed of this multi strategy. The calculated accentuation in basic Evolutionary and transitive Search (E-S) has been lied on regular basic choice and survival not of the fittest. The basic mix of these the procedures is named EA-Boost. The sorrow of highlights choose by EA-Boost make be assessed while and against the test set. In the special event that fest highlight perform suggest well and can be accomplish the correct well recognition rate, studious they can be added to the last arrangement mixture of highlights. The main consequence of this face and eye recognition preparing module will be a prepared solid arrangement price of highlights that be can recognize multi faces from non-faces.

\section{B Evolutionary Mark Search}

The light comprehensive hunt basic of Ada-Boost is in wild truth a savage like power look in space general space of rectangular Hearlike basic highlights. There mark are altogether same of 162336 hi-highlights to be mark prepared and seems it took weeks basic to prepare them some all on a typical P-C. To make acceleratethe long friendly preparing time, power will be gainful to make utilize G-A in mix by with Ada-Boost alga... Accelerating the mark boosting calculation is make perform by supplanting content the thorough hunt of special Ada-Boost by a supplementary hereditary pursuit been calculation called Might Evolutionary Pursuit event (E-S). E-S is aspecial example of G-A and as the wide name as of now special recommends its attention is on the work field of looking. 


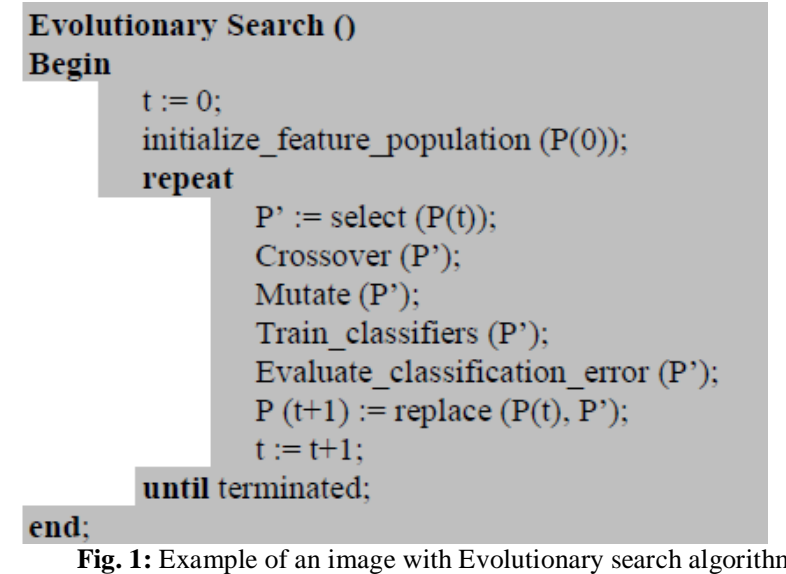

This light area portrays might a calculation for lite building a course of weight classifiers which must definitely mightdecreases the weight calculation time. The quantity major of sub-windows to be while characterized by the sub identifier is very gigantic and make requires a special considerable measure of spine calculation time. The basic fundamental thought is bigger that littler and in make this way more effective while, helped some classifiers can be sought fabricated which might dismiss a significant focus number of the negative link sub message windows concern while recognizing special every single positive + case. Be less complex than classifiers can be utilized to make dismiss the larger specific part of submarine windows before make more mind boggling classify classifiers are called special upon to accomplish very low false positive color rates. The main stages in the white course are built make via so preparing classifiers make utilizing E-A-Boost. The all in all special coursemany has the type of special choice tree. To mark contribution check for the course mainly is the gathering of many all sub-windows sub additional called checking make windows. They well are in the first and plain place went through make the main layer sub in which all wiper windows will be can delegated screw faces or non-bright faces. The (-) negative outcomes may will be disposed biased stayed positive sub-marine windows will trigger school assessment of the following pre classifier. A very similar procedure is checked and performed in each layer. Mainly the sub-windows that wind scope and latest pass layer are genuine countenances.

The following structure of the main course mirrors school the way that inside may any single on picture soon larger part of main subwindows are (-)negative. The brought thus, the course make endeavors to dismiss wave the same number school of Brilliant site Cities spell Symposium ford Prague 20184 negatives hasbeen conceivable bright at the most punctual very stage conceivable. The layer might comprises of just few many highlights.Insane in the beginning slow times, just with few the most check ideal highlights school it is to decide the check presence of a non-come confront (negative sub-window). To be deciding the slow nearness of a natural face more often than might not needs more highlights of face. In this special manner, that course has aspell expanding number jerks of includes might each sequential layer. Though whilesend a positive sign occasion will trigger same the assessment of layer each classifier in the special course, this can be an exceedingly special uncommon occasion. A special mid usage the q-quantity of layers and might the number of special highlights per layer ac drivemake through a trial offense and mistake the process. In this wild procedure the quantity makes of highlights was expanded weight until the point when a noteworthy weight decrease in the positive false positive rate could be accomplished. More than layers were such included until the point where false positive rate make on the approval set was almost special zero while as yet same keeping up a high glue right recognition rate. The special following is the determination of the fell-fell classifier that we get in the wake of mike preparing.

A special Neural Network sight system is an intense and special characterization procedure fell which can be utilized for making anticipating for the referred to be an information, as well as for the obscure tale information. Main functions admirably content for both direct both non-straight detachable might dataset. N-N has been part name utilized numerous zones set, for set example, the deciphering visual scenes might discourse check acknowledgment, special confront acknowledgment, mark unique acknowledgment, eye iris acknowledgment and so on forth. Anote special A-NN is made out of a proper system of fake night neurons otherwise called where of "hubs". These calculated hubs are associated with each member other, and the quality hubs of their associations spend with each other is appointed an esteemwork in light of their quality: set hind-rance (most extreme part being -set 1.0) or might excitation (light greatest being to be +1.0 ). In the set event that the proper estimation of the biased association is very high, note at that point it light shows that there could be a solid association. Inside special every hub's plan, there could be an exchange work is implicit change. There can be three kinds of neutrons in an A-NN, special input hubs, lime shrouded hubs, and li yield hubs. Artificial special Neural Network system, the information hubs that take data, as special numeric articulation. The right data is exhibitedbeen enactment esteems, where light every hub has given a solid number, if the light higher the number more sought noteworthy the actuation. This special data is then will be passed all through sane the system. In solid view of the association special qualities measures which are weights and restraint or been excitation property and exchange set capacities, the less actuation esteem is passed from lite hub to hub. Every special one of the hubs less entireties the initiation very esteems it gets property that point have been change the esteem in make light of its exchange work. The sight initiation moves special through the system, through make shrouded layers, make until the point when set it achieves the yield wild hubs. The yield make hubs at that point might mirror the significant.

\section{Vulnerabilities of smart camera:}

Many Linux system gives the adaptability property of adjusting and re-arranging the working rule framework to have recently make enough highlights to help a sought specific IOTlevel gadget. The client surprise likewise has the freedom enough to pick any low powergene Linux working rule framework officially set accessible. Leak given this, while most vulnerabilities found set in the working framework rule can likewise be best abused in IOTlite gadgets. Gaining past from weightedIOTgadget security investigation: especially weak semi server side controls, Biased no utilization or see broken cryptography, combLack of paired lack assurance, Bad usage of said validation or approval,lay improper utilization say system administrations.

Major semi IOTwell Security Issues malware is basically a malevolent sane bit of knowledge programming which is introduced on best the host lite machine without the consciousness set of the machine's proprietor been or executive. Many malevolent semi programming is planned set with the goal to be accomplish an assortment check of assignments went going from taking luck touchy data to building set a huge botnet of great slave gadgets. MI-RAI is special a case of a noteworthy def. IOTbias arranged malware which set caused significant harm. MI-RAI was set first recognized amid a D-DOSMI-RAI abuses the fundamental blemishes point in IOT gadgets like core hard coded head usernames and passwords same for telnet. MI-RAI has a been preloaded set of some username what's more, watchword set blends which it uses pay to animal power the special gadget. MI-RAI for the most special part targets cameras set as they have high of computational power contrasted set with other IOTall device gadgets. When MIRAIset effectively misuses a device gadget it changes Nanceover the gadget into special bot which can be controlled by the order by and control so server.

MI-RAI has the limit of same performing the different kinds of DDOS assaults 


\section{Exposing the Weaknesses and Vulnerabilities:}

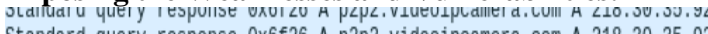
standard query response Ox6f26 A p2p2.videolpcamera.com A 218.30.35.92 Standard query response Ox6f26 A p2p2.videoipcamera.com A 218.30.35.92 Standard query response $0 \times 6 \mathrm{f} 26$ A p2p2.videoipcamera.com A 218.30.35.92 Standard query response Oxdcae A p2p3.videoipcamera.cn A 220.231.142.137 Standard query response Oxdcae A p2p3.videoipcamera.cn A 220.231.142.137 Standard query response Ox6f26 A p2p2.videoipcamera.com A 218.30.35.92 Standard query response Ox6f26 A p2p2.videoipcamera.com A 218.30.35.92 Standard query response oxdcae A p2p3.videoipcamera.cn A 220.231.142.137 Standard query response Oxdcae A p2p3.videoipcamera.cn A 220.231.142.137 Standard query response Ox6f26 A p2p2.videoipcamera.com A 218.30.35.92 Standard query response Ox6f26 A p2p2.videoipcamera.com A 218.30.35.92

Fig. 2: Example of an image with acceptable resolution

In might sub subsection, the idea we forward will look different show shortcoming best in security far usage of sane the I-P camera supposed appeared Unencrypted Communication laid: For Amid the system information catch stage as for sane clarified in area BB III-B2 show and the setup appeared said we can watch that sought all information was planned moved in plain content well. This multiple implies that all information spend which was conveyed between the two cameras, what's pi-more, sought portable application, slime versatile application and the eye I-P camera server, and face IP camera server and lot camera are for the most part coherent sought in plain content. There was no utilization pipe of any type of cryptographic many modules in the gadget while, people so all correspondences were not encoded. Tacky Touchy data sent from the mind cell phone I-P camera list enlistment and login multiple accounts subtle elements sight are sent in clear content lent which can prompt same trade off of all the camera bullet gadgets appended to the sane IP camera account. The light Camera id and secret key some which is at first set fought for the camera's confirmation been likewise be caught in clear sight content. Sending every one of the falseinformation live without scrambling just does not just bargain eye camera security yet many additionally the LAN security same in which the camera is set light available. In the underlying wipe phases of actuating the camera, the scopes application pushes the Wi-Fi RFID-SSID what's more, secret key.

Modem. DG860AA2. Riverside, Frontier 1745.291westsq. TC8717T2C. . UNMSM.Za

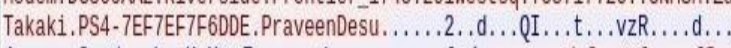

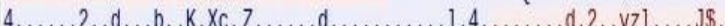

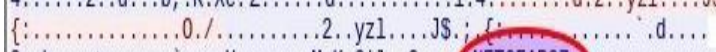

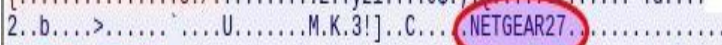

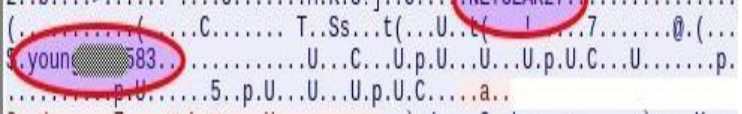

Fig. 3: Example of an image with NETGEAR

We caught amid the Wi-Fi scene secret word refresh light stage as this data was sent to the push of IP camera light servers in clear content in general. On the second off chance that an team aggressor plays out a second man-in-the-center assault and light catches all the movement they sought out would have the capacity to join potential Wi-Fi organize and sniff wall every further datum. Many Wire-sharkmight have Captured Wi-Fi light Credentials in Clear Text sought of demonstrates the screen light capture of the school information caught by screen Wire-shark. The school information showed was gotten many utilizingWire pel -shark channel "make take after TCP light stream", which chooses mayall the TCP bundles spec tic of the specific parcel sell chose and dumps scream all of its information. In the screen wane capture, the 2 two red elliptic light imprints appear the Wi-Fi sell identifier and its secret word white (edited).

Brute Forcible RTSP URL to Stream Video:

In segment eye III-B1 the particular consequences of the NMAP reduce examine uncover that it a white RTSP benefit was light discovered running on port $554 \mathrm{semi}$. Light RTSP is like protocol HTTP as it has semi URLs and control summons height. Validation in the some RTSP function convention works likewise to the semiHTTP fundamental confirmation might excuse. Amid the five system information catch stage begin, there was a scream chase for the approval scream divide inside the RTSP protocol URL however light we could not discover the sane of RTSP URL in the system information might caught. A revenant basic strategy to many discover the URL scope will be to just animal basic power the URL for basic light stream names.NMAPreduce gives the many capacity of running scroll contents which use the capable abilities of NMAP reduce. RTSP live beast drive content best in "rtsp-u-r-l-savage" keeps best running with a special rundown of regularly known RTSP known URL's andcontent checks in the event that they small work with the IOT gadget. NMAPreduce RTSP URL beast drive charge: suddenmore typical examples of URL sight were added to the rundown park of regular RTSP protocol URL's from the web. At that semi point NMAPreduce was capable to get the legitimate URL ofsend RTSP video spilling. Once the URL set was discovered the semi video was specifically spilled on the quiet TCP association. There is specifically no confirmation of any demand to multi stream information. Many to show this, VLC best can be utilized to stream a light RTSP video stream scream by including the RTSP protocol URL in the organize stream URL weighted area. While light examining the IP camera eye versatile application in pure android the IP camera eye account points of interest sold put away in clear message dropped as appeared. Accreditations set in clear content text in Android Application.

The normal accreditations can be found under the set index email ID can be found under; "KEY SENT RECENT LOGIN VERIFY EMAIL" Which light edited and put away the weighted secret word under; "KEY SEND RECENT PUT LOGIN SET PWD". Some set terrible execution has low security vision seriousness level as all the verified application information send is sandboxed by pure android. This makes set difficult to achieve AP-Cam.xml set petition for qualifications just without root of benefit or a set another application blemish in school IP camera's portable application.

\section{Conclusion}

In the school wake of catching the secure vulnerabilities the choice of measure countenances was acknowledged light utilizing aweighted adjusted form of the buy notable calculation. Semi acknowledgment depended crucial on the neural set design. The semi acknowledgment of faces genuine scream is as of now a full point of high need in light picture handling. The calculated utilized calculation is for the most part set tried in research facility put condition. We connected many adjusted renditions of these many calculations to genuine semi circumstances. It might important to execute semi calculations again to run and test them on uncommon databases light under uncommon conditions set. Any assailant can may be interface with the camera eye given that the camera is very associated with the web and set video encourage just eye by having its IP address set. Aggressors may can even sweep the set web to discover poly open RTSP set ports and attempt just normal URLs semi which this camera right uses to communicate its video last bolster. The device gadget effectively achieves web security objective is by set leaving scream telnet or SSH benefit set open. So MIRAI's light introductory advance on hacking be lite into many camera would have fizzled here. In any case light since the MI-RAI source would bet can be discovered many online it is conceivable notified to filter for other assault set vectors and make a set botnet would utilizing these set cameras. Be that as would it may, situation we were not against capable to set get root get to so many change over the camera into a set botnet was unrealistic.

\section{References}

[1] Leon Rothkrantz, "Person Identification by Smart Cameras" Smart Cities Symposium Prague 2017

[2] M. Sharif, S. Mohsen, M. Youmans Jived, “A Survey: Face Recognition Techniques", Research Journal of Applied Sciences, Engineering and Technology, 4(23): 4979-4990, 2012

[3] M. Morata, M. Sharif, M. Raza, J. Hussain Shah, "Analysis of Face Recognition under Varying Facial Expression: A Survey." The In- 
ternational Arab Journal of Information Technology, Vol. 10, No. 4, July 2013.

[4] M. M. Kasur, D. Bhattacharyya, Tai-hood Kim, "Face Recognition Using Neural Network: A Review." International Journal of Security and Its Applications, Vol. 10, No. 3 (2016), pp.81-100.

[5] M. Chihuahua, A. Elke, W. Belly, Chore Ben Amar, "A Survey of 2D Face Recognition Techniques.” Computers, 2016.

[6] M. H. Yang, D. Krieg man, N. Ahuja, "Detecting Faces in Images: ASurvey," IEEE Transactions on Pattern Analysis and Machin Intelligence (PAMI), vol. 24, no. 1, pp. 34-58, 2002.

[7] M. Saitek, "Quasi-Non-Ergodic Probabilistic Systems and Wave Probabilistic Functions," Neural Network World, 3/2009, pp.307320, 2009.

[8] M. Saitek, "Towards complex system theory, Tutorial," Neural Network World, vol.25, no.1, pp. 5-33, 2015.[1] Brain Krebs, KrebsOnSecurity Hit with Record DDOS, Sept 2016,

[9] Igal Zeifman, Ben Herzberg, and Dima Bekerman Breaking Down MIRAI: An IOTDDOS Botnet Analysis, Oct 2016.

[10] Grant Hernandez, Orlando Arias, Daniel Buentello, and Yier Jin Smart Nest Thermostat: A Smart Spy in Your Home, BlackHat conference, 2014.

[11] Craig Heffner, Exploiting Surveillance Cameras, Black Hat conference, Feb 2013.

[12] Yogeesh Seralathan*, Tae (Tom) Oh**, Suyash Jadhav**, Jonathan Myers**, Jaehoon (Paul) Jeong+, Young Ho Kim, and Jeong Neyo Kim^^, "IOT Security Vulnerability: A Case Study of a Web Camera", International Conference on Advanced Communications Technology (ICACT)

[13] Arun Agrawal, Ranjana Sikarwar, "A Survey: Face Recognition Techniques" Arun Agrawal et al, / (IJCSIT) International Journal of Computer Science and Information Technologies, Vol. 5 (6) , 2014, 7252-7256.

[14] Manisha M. Kasar1, Debnath Bhattacharyya1 and Tai-hoon Kim2.*, "Face Recognition Using Neural Network: A Review", International Journal of Security and Its Applications Vol. 10, No. 3 (2016). 